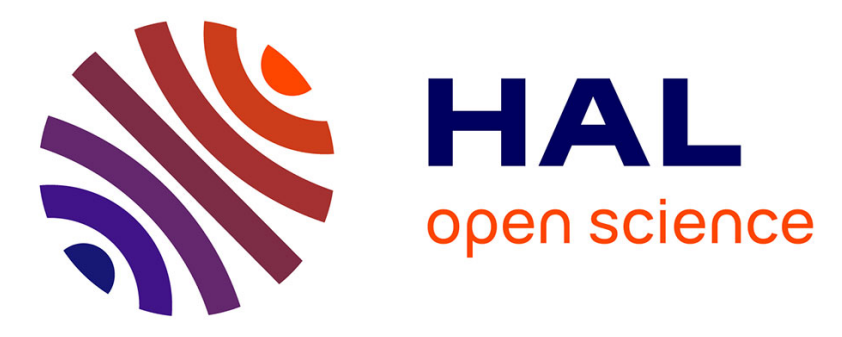

\title{
Understanding the difference between prox and complementarity formulations for simulation of systems with contact
}

Thorsten Schindler, Binh Nguyen, Jeff Trinkle

\section{- To cite this version:}

Thorsten Schindler, Binh Nguyen, Jeff Trinkle. Understanding the difference between prox and complementarity formulations for simulation of systems with contact. IEEE/RSJ International Conference on Intelligent Robots and Systems, Sep 2011, San Fransisco, United States. 10.1109/IROS.2011.6094779 . hal-01309169

\section{HAL Id: hal-01309169 \\ https://hal.science/hal-01309169}

Submitted on 29 Apr 2016

HAL is a multi-disciplinary open access archive for the deposit and dissemination of scientific research documents, whether they are published or not. The documents may come from teaching and research institutions in France or abroad, or from public or private research centers.
L'archive ouverte pluridisciplinaire HAL, est destinée au dépôt et à la diffusion de documents scientifiques de niveau recherche, publiés ou non, émanant des établissements d'enseignement et de recherche français ou étrangers, des laboratoires publics ou privés. 


\section{Understanding the difference between prox and complementarity formulations for simulation of systems with contact}

\author{
Thorsten Schindler \\ INRIA Grenoble - Rhône-Alpes \\ thorsten.schindler@mytum.de
}

\author{
Binh Nguyen \\ Rensselaer Polytechnic Institute \\ nguyeb2@cs.rpi.edu
}

\author{
Jeff Trinkle \\ Rensselaer Polytechnic Institute \\ trink@cs.rpi.edu
}

\begin{abstract}
To plan a robotic task involving intermittent contact, such as an assembly task, it is helpful to be able to simulate the task accurately and efficiently. In the past ten years, the prox formulation of the equations of motion has arisen as a competitive alternative to the well-known linear and nonlinear complementarity problem (LCP and NCP) formulations. In this paper, we compare these two formulations, showing through a set-based argument that the formulations are equivalent. Second, we provide simple examples to compare the most common approaches for solving these formulations. The prox formulation is solved by fixed-point iteration while the complementarity formulation is solved by a pivoting scheme, known as Lemke's algorithm. The well-known paradox of Painlevé is used in a case where two solutions exist to illustrate that the fixed-point scheme can fail while the pivoting scheme will succeed.
\end{abstract}

\section{INTRODUCTION}

In robotics, we are interested in the analysis of tasks involving intermittent contact, e.g., product assembly and optimal control of walking machines. The equations of motion of such kinds of applications can be written as a system of differential equations with set-valued constraints

$$
\begin{gathered}
\dot{\boldsymbol{q}}=\boldsymbol{Y}(\boldsymbol{q}) \boldsymbol{u}, \\
\boldsymbol{M}(\boldsymbol{q}) \dot{\boldsymbol{u}}=\boldsymbol{h}(\boldsymbol{q}, \boldsymbol{u}, t)+\boldsymbol{W}(\boldsymbol{q}) \boldsymbol{\lambda}, \\
(\boldsymbol{q}, \boldsymbol{u}, \boldsymbol{\lambda}, t) \in \mathcal{N} .
\end{gathered}
$$

We refer to $t$ as the time, to $\boldsymbol{q}$ as the configuration of the system and to $\boldsymbol{u}$ as its velocity. The elements of $\boldsymbol{\lambda}$ are rigid contact reactions e.g. between the robot's hand and tool or between feet of a walker and floor. Rigid means that these contact reactions are represented by constraints: the set $\mathcal{N}$ restricts the possible values such that there is no interpenetration and that friction forces maximize power dissipation at sliding contacts. As a consequence, the acceleration function $\dot{u}$ exhibits jump discontinuities over time. Further, impact equations have to be added in case of closing contacts, e.g. during stepping and grasping. The vector $\boldsymbol{h}$ contains all smooth external, internal and gyroscopic forces, which are functions of $\boldsymbol{q}, \boldsymbol{u}$ and explicitly of $t$. It also includes compliant contacts. The symmetric and positive definite mass matrix $\boldsymbol{M}$, the velocity kinematic map $\boldsymbol{Y}$, and the constraint Jacobian $\boldsymbol{W}$ depend only on $\boldsymbol{q}$.

In most physics engines used in robotic simulations, a linearized complementarity formulation has been used to represent the constraints $\mathcal{N}$, and a pivoting algorithm has been used to solve the equations of motion directly. The prox formulation, first stated at the beginning of the 1990s [1], is said to possess better numerical properties in practical simulation applications such as robotic manipulation tasks, the operation of continuously variable transmissions and electrical circuit breaker systems [2], [3], [4]. We analyze the prox and complementarity formulations showing their mathematical equivalence. Further, we study the qualitative performance of typical solvers through two simple examples.

\section{COMPLEMENTARITY FORMULATION}

Joints of a robot system are represented by so-called bilateral constraints

$$
B_{C}:=\left\{\left(g_{B}, \lambda_{B}\right) \in \mathbb{R} \times \mathbb{R} \mid g_{B}=0\right\} .
$$

Each such joint is physically enforced (i.e., $g_{B}$ is held to zero) by a contact force $\lambda_{B}$. Note that the bilateral condition (4) is expressed at configuration level, but it can also be written at the velocity level when $g_{B}=0$, by replacing $g_{B}=0$ in (4) with $\dot{g}_{B}=0$. Similarly the condition can be written at the acceleration level when $\dot{g}_{B}=g_{B}=0$, by replacing $g_{B}=0$ by $\ddot{g}_{B}=0$.

Unilateral contacts can form and detach intermittently. Such behavior is represented by SIGNORINI-FICHERAconditions [2]

$$
U_{C}:=\left\{\left(g_{U}, \lambda_{U}\right) \in \mathbb{R} \times \mathbb{R} \mid 0 \leq g_{U} \perp \lambda_{U} \geq 0\right\}
$$

where $g_{U}$ denotes the normal distance and the symbol $\perp$ implies orthogonality (i.e $g_{U} \lambda_{U}=0$ ) to the contact force $\lambda_{U}$. These conditions can also be formulated at the velocity or acceleration level as described for $g_{B}$. The respective forcedisplacement laws are shown in Figs. 1(a) and 1(b).

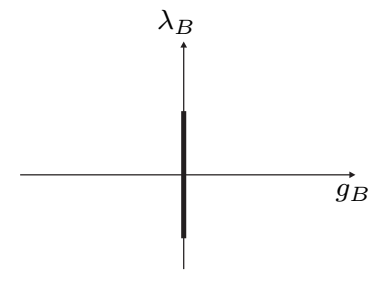

(a) Bilateral constraint

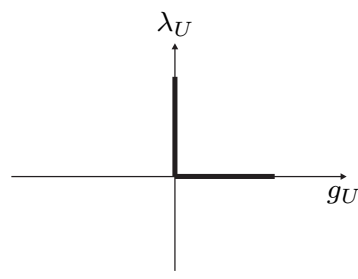

(b) Unilateral constraint
Fig. 1. Force laws for bilateral and unilateral contacts.

For both bi- and unilateral constraints, dry friction can be considered. In order to establish CoulOMB's law, the force of a single contact is decomposed in a positive component $\lambda_{N} \in\left\{\lambda_{B}, \lambda_{U}\right\}$ normal to the contact tangent plane and 
tangential components $\boldsymbol{\lambda}_{T}$ in the tangent plane. When the relative velocity $\dot{\boldsymbol{g}}_{T}$ at the contact point is nonzero, COULOMB's friction law is given as follows:

$$
\begin{aligned}
T_{C}\left(\lambda_{N}\right):=\left\{\left(\dot{\boldsymbol{g}}_{T}, \boldsymbol{\lambda}_{T}\right) \in \mathbb{R}^{2} \times \mathbb{R}^{2}\right. \\
\dot{\boldsymbol{g}}_{T}=\mathbf{0} \Rightarrow\left\|\boldsymbol{\lambda}_{T}\right\| \leq \mu\left|\lambda_{N}\right|, \\
\left.\dot{\boldsymbol{g}}_{T} \neq \mathbf{0} \Rightarrow \boldsymbol{\lambda}_{T}=-\frac{\dot{\boldsymbol{g}}_{T}}{\left\|\dot{\boldsymbol{g}}_{T}\right\|} \mu\left|\lambda_{N}\right|\right\}
\end{aligned}
$$

where $\mu>0$ is the coefficient of friction. In the degenerate case defined by $\mu=0$ or $\lambda_{N}=0$, these conditions are not needed in the problem formulation. If the contact is sticking (i.e. $\dot{\boldsymbol{g}}_{T}=0$ ) then in (6), $\dot{\boldsymbol{g}}_{T}$ is replaced by $\ddot{\boldsymbol{g}}_{T}$ to calculate forces. One can easily show that in the non-degenerate cases $T_{C}\left(\lambda_{N}\right)$ equals the complementarity formulation:

$$
\begin{aligned}
T_{\sigma}\left(\lambda_{N}\right) & :=\left\{\left(\dot{\boldsymbol{g}}_{T}, \boldsymbol{\lambda}_{T}\right) \in \mathbb{R}^{2} \times \mathbb{R}^{2}, \sigma \in \mathbb{R} \mid\right. \\
\mathbf{0} & =\mu\left|\lambda_{N}\right| \dot{\boldsymbol{g}}_{T}+\sigma \boldsymbol{\lambda}_{T}, \\
0 & \left.\leq \sigma \perp \mu\left|\lambda_{N}\right|-\left\|\boldsymbol{\lambda}_{T}\right\| \geq 0\right\} .
\end{aligned}
$$

In the planar case, CoulomB's law is depicted in Fig. 2.

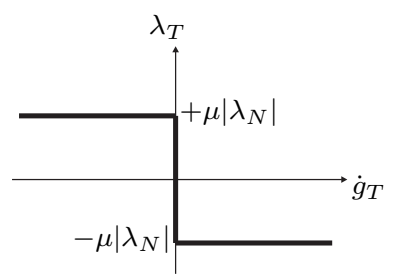

Fig. 2. Planar COUlomB friction. The vertical part of the graph reveals the set-valued nature of the constraint, i.e., when sliding velocity is zero, the friction force may take on any value in $\left(-\mu\left|\lambda_{N}\right|,+\mu\left|\lambda_{N}\right|\right)$.

Finally, the complementarity formulation of a system of bodies in three dimensions with bilateral and unilateral constraints with COUlOMB friction is given by (1)-(3) and all of the equations and inequalities in (4), (5) and (7). These equations represent the instantaneous equations of motions whose form is known as a nonlinear differential complementarity problem (DCP). Trajectories of the system constructed during simulation are obtained by solving a timesequence of nonlinear complementarity problems (NCPs). These can be solved reliably with the PATH solver [5], [6].

\section{FORMULATION WITH PROX FUNCTION}

A perhaps more appropriate formulation can be obtained using convex analysis. For $\boldsymbol{x} \in \mathbb{R}^{n}$ and $n \in \mathbb{N}$, we define

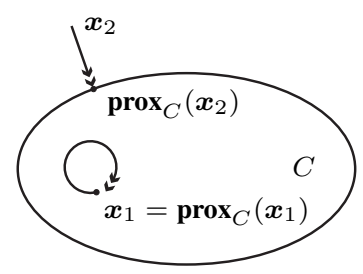

Fig. 3. prox function for a convex set.

the closest point, i.e. the proximal point, to a given convex set $C \subset \mathbb{R}^{n}$ :

$$
\operatorname{prox}_{C}(\boldsymbol{x})=\arg \min _{\boldsymbol{x}^{*} \in C}\left\|\boldsymbol{x}-\boldsymbol{x}^{*}\right\|
$$

In Fig. 3, it is illustrated that the proximal point is the point itself when it is in $C$ (cf. $\boldsymbol{x}_{1}$ ) or the closest point on the boundary of $C$ otherwise (cf. $\boldsymbol{x}_{2}$ ). The contact conditions can be formulated as:

$$
\begin{aligned}
& B_{P}:=\left\{\left(g_{B}, \lambda_{B}\right) \in \mathbb{R} \times \mathbb{R} \mid\right. \\
& \left.f_{B}\left(\lambda_{B}, g_{B}\right):=\lambda_{B}-\operatorname{prox}_{C_{B}}\left(\lambda_{B}-r g_{B}\right)=0\right\}, \quad(9), \quad \\
& U_{P}:=\left\{\left(g_{U}, \lambda_{U}\right) \in \mathbb{R} \times \mathbb{R} \mid\right. \\
& \left.f_{U}\left(\lambda_{U}, g_{U}\right):=\lambda_{U}-\operatorname{prox}_{C_{U}}\left(\lambda_{U}-r g_{U}\right)=0\right\}, \\
& T_{P}\left(\lambda_{N}\right):=\left\{\left(\dot{\boldsymbol{g}}_{T}, \boldsymbol{\lambda}_{T}\right) \in \mathbb{R}^{2} \times \mathbb{R}^{2} \mid\right. \\
& \left.\boldsymbol{f}_{T}\left(\boldsymbol{\lambda}_{T}, \dot{\boldsymbol{g}}_{T}\right):=\boldsymbol{\lambda}_{T}-\operatorname{prox}_{C_{T}\left(\lambda_{N}\right)}\left(\boldsymbol{\lambda}_{T}-r \dot{\boldsymbol{g}}_{T}\right)=\mathbf{0}\right\}
\end{aligned}
$$

where the corresponding convex sets are specified by:

$$
\begin{aligned}
C_{B} & =\mathbb{R}, \\
C_{U} & =\{x \in \mathbb{R} \mid x \geq 0\}, \\
C_{T}(y) & =\left\{\boldsymbol{x} \in \mathbb{R}^{2}|\|\boldsymbol{x}\| \leq \mu| y \mid\right\}
\end{aligned}
$$

with $y \in \mathbb{R}$. The equations are given at the level of the lowest-order derivatives, configuration in normal directions $N \in\{B, U\}$ and velocity in tangential directions. Higherorder derivative formulations can be obtained simply by increasing the number of dots over the $\boldsymbol{g}$ 's. An independent auxiliary parameter $r \in \mathbb{R}^{+}$appears in (9). This parameter is used to match units in the equations, but more importantly, its value strongly affects the stability and rate of convergence when finding a solution by fixed-point iteration. It is also important to notice that the solution set of (9) is nonsmooth. Typical spatial plots of $f_{B}, f_{U}$ and $f_{T}$ are given in Fig. 4 for $r=1.0$. The solution set is the intersection of the dark surface with the light plane. Notice the corner(s) for $f_{U}$ and $\boldsymbol{f}_{T}$. In the tangential cases, $\mu=0.1$ and $\lambda_{N}=1$ have

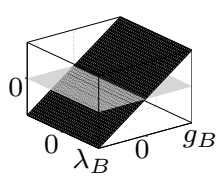

(a) Bilateral

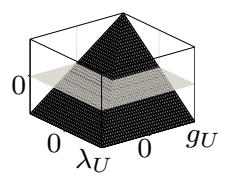

(b) Unilateral

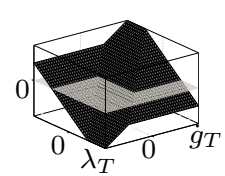

(c) Friction
Fig. 4. prox functions for contacts and friction with $r=1.0$.

been used. The intersection with the zero-constraint and the solution sets are reminiscent of the force laws from Figs. 1 and 2. Mathematically this is proved in Section IV.

\section{EQUIVALENCE BETWEEN COMPLEMENTARITY AND PROX}

Complementarity and prox formulations represent the same constraints

$$
B_{C}=B_{P}, U_{C}=U_{P}, T_{C}=T_{P}
$$

as has been shown in [1] using variational equations. Therefore, numerical solution strategies can be devised to make 
use of their advantages while avoiding their disadvantages. We show the equivalences for the case of the lowest-order derivatives starting basically from the set definitions like in [7], [8] but proceeding more directly.

\section{Bilateral constraint}

Starting with the prox formulation $\left(g_{B}, \lambda_{B}\right) \in B_{P}$, it is obvious that the argument $\left(\lambda_{B}-r g_{B}\right)$ of $\operatorname{prox}_{C_{B}}$ is always an element of the corresponding convex set $C_{B}=\mathbb{R}$. This yields:

$$
0=\lambda_{B}-\lambda_{B}+r g_{B}
$$

and finally $g_{B}=0$ as well as $\left(g_{B}, \lambda_{B}\right) \in B_{C}$.

In the complementarity formulation, it is $\lambda_{B} \in \mathbb{R}$ and $g_{B}=0$. The relations both $\lambda_{B}=\operatorname{prox}_{C_{B}}\left(\lambda_{B}-r g_{B}\right)$ and $\left(g_{B}, \lambda_{B}\right) \in B_{P}$ are obvious.

\section{Unilateral constraint}

Starting with the prox formulation $\left(g_{U}, \lambda_{U}\right) \in U_{P}$, one has to distinguish two cases.

1) $\lambda_{U}-r g_{U} \in C_{U}$ or $\lambda_{U}-r g_{U} \geq 0$ :

This yields $0=\lambda_{U}-\lambda_{U}+r g_{U}$ and $g_{U}=0$ as well as $\lambda_{U} \geq 0$.

2) $\lambda_{U}-r g_{U} \notin C_{U}$ or $\lambda_{U}-r g_{U}<0$ :

This yields $\lambda_{U}=\operatorname{prox}_{C_{U}}\left(\lambda_{U}-r g_{U}\right)=0$ and $g_{U}>0$.

This is a representation of the unilateral corner law by a complementarity formulation $\left(g_{U}, \lambda_{U}\right) \in U_{C}$.

In a complementarity formulation $\left(g_{U}, \lambda_{U}\right) \in U_{C}$, there are two cases for the branches of the unilateral corner law.

1) $g_{U}=0$ with $\lambda_{U} \geq 0$ :

This yields $\lambda_{U}-r g_{U}=\lambda_{U} \geq 0$ or $\lambda_{U}-r g_{U} \in C_{U}$ and the validity of the prox formulation $\left(g_{U}, \lambda_{U}\right) \in U_{P}$ in the specific case.

2) $g_{U}>0$ and $\lambda_{U}=0$ :

This yields $\lambda_{U}-r g_{U}<0$ or $\lambda_{U}-r g_{U} \notin C_{U}$ and the validity of the prox formulation $\left(g_{U}, \lambda_{U}\right) \in U_{P}$ in the specific case.

\section{Coulomb friction}

Let $\mu \geq 0$ and $\lambda_{N} \in \mathbb{R}$.

Starting with the prox formulation $\left(\dot{\boldsymbol{g}}_{T}, \boldsymbol{\lambda}_{T}\right) \in T_{P}\left(\lambda_{N}\right)$, one has to distinguish two cases.

1) $\boldsymbol{\lambda}_{T}-r \dot{\boldsymbol{g}}_{T} \in C_{T}\left(\lambda_{N}\right)$ or $\left\|\boldsymbol{\lambda}_{T}-r \dot{\boldsymbol{g}}_{T}\right\| \leq \mu\left|\lambda_{N}\right|$ : This yields $\mathbf{0}=\boldsymbol{\lambda}_{T}-\boldsymbol{\lambda}_{T}+r \dot{\boldsymbol{g}}_{T}$ and $\dot{\boldsymbol{g}}_{T}=\mathbf{0}$. One has $\left\|\boldsymbol{\lambda}_{T}\right\| \leq \mu\left|\lambda_{N}\right|$ and finally $\left(\dot{\boldsymbol{g}}_{T}, \boldsymbol{\lambda}_{T}\right) \in T_{C}\left(\lambda_{N}\right)$.

2) $\boldsymbol{\lambda}_{T}-r \dot{\boldsymbol{g}}_{T} \notin C_{T}\left(\lambda_{N}\right)$ or $\left\|\boldsymbol{\lambda}_{T}-r \dot{\boldsymbol{g}}_{T}\right\|>\mu\left|\lambda_{N}\right|$ :

This yields:

$$
\begin{aligned}
\boldsymbol{\lambda}_{T} & =\frac{\boldsymbol{\lambda}_{T}-r \dot{\boldsymbol{g}}_{T}}{\left\|\boldsymbol{\lambda}_{T}-r \dot{\boldsymbol{g}}_{T}\right\|} \mu\left|\lambda_{N}\right| \\
& =-\underbrace{\frac{r\left\|\dot{\boldsymbol{g}}_{T}\right\|}{\left\|\boldsymbol{\lambda}_{T}-r \dot{\boldsymbol{g}}_{T}\right\|-\mu\left|\lambda_{N}\right|}}_{k} \frac{\dot{\boldsymbol{g}}_{T}}{\left\|\dot{\boldsymbol{g}}_{T}\right\|} \mu\left|\lambda_{N}\right| .
\end{aligned}
$$

If one were in the case $\dot{\boldsymbol{g}}_{T}=\mathbf{0}$, this would be a contradiction because

$$
\left\|\boldsymbol{\lambda}_{T}\right\|>\mu\left|\lambda_{N}\right| \wedge \boldsymbol{\lambda}_{T}=\frac{\boldsymbol{\lambda}_{T}}{\left\|\boldsymbol{\lambda}_{T}\right\|} \mu\left|\lambda_{N}\right| .
$$

One has to discuss the case $\dot{\boldsymbol{g}}_{T} \neq \mathbf{0}$. Then, the contact is sliding and we must have $\left\|\boldsymbol{\lambda}_{T}\right\|=\mu\left|\lambda_{N}\right|$. Comparing the expressions in (15), one sees that $k$ must equal 1 . Therefore, the only solution is given by $\boldsymbol{\lambda}_{T}=-\frac{\dot{\boldsymbol{g}}_{T}}{\left\|\dot{\boldsymbol{g}}_{T}\right\|} \mu\left|\lambda_{N}\right|$ and $\left(\dot{\boldsymbol{g}}_{T}, \boldsymbol{\lambda}_{T}\right) \in T_{C}\left(\lambda_{N}\right)$.

If one has a representation by a complementarity formulation $\left(\dot{\boldsymbol{g}}_{T}, \boldsymbol{\lambda}_{T}\right) \in T_{C}\left(\lambda_{N}\right)$, again there are two cases.

1) $\dot{\boldsymbol{g}}_{T}=\mathbf{0}$ and $\left\|\boldsymbol{\lambda}_{T}\right\| \leq \mu\left|\lambda_{N}\right|$ :

This yields $\boldsymbol{\lambda}_{T}-r \dot{\boldsymbol{g}}_{T} \in C_{T}\left(\lambda_{N}\right)$ and trivially $\left(\dot{\boldsymbol{g}}_{T}, \boldsymbol{\lambda}_{T}\right) \in T_{P}\left(\lambda_{N}\right)$.

2) $\dot{\boldsymbol{g}}_{T} \neq \mathbf{0}$ and $\boldsymbol{\lambda}_{T}=-\frac{\dot{\boldsymbol{g}}_{T}}{\left\|\dot{\boldsymbol{g}}_{T}\right\|} \mu\left|\lambda_{N}\right|$ :

This yields:

$$
\begin{aligned}
\left\|\lambda_{T}-r \dot{\boldsymbol{g}}_{T}\right\| & =\left\|-\frac{\dot{\boldsymbol{g}}_{T}}{\left\|\dot{\boldsymbol{g}}_{T}\right\|} \mu\left|\lambda_{N}\right|-r\right\| \dot{\boldsymbol{g}}_{T}\left\|\frac{\dot{\boldsymbol{g}}_{T}}{\left\|\dot{\boldsymbol{g}}_{T}\right\|}\right\| \\
& =\mu\left|\lambda_{N}\right|+r\left\|\dot{\boldsymbol{g}}_{T}\right\|>\mu\left|\lambda_{N}\right|
\end{aligned}
$$

and $\boldsymbol{\lambda}_{T}-r \dot{\boldsymbol{g}}_{T} \notin C_{T}\left(\lambda_{N}\right)$. Finally:

$$
\begin{aligned}
\boldsymbol{\lambda}_{T} & =-\frac{\dot{\boldsymbol{g}}_{T}}{\left\|\dot{\boldsymbol{g}}_{T}\right\|} \mu\left|\lambda_{N}\right| \\
& =-\frac{\mu\left|\lambda_{N}\right|+r\left\|\dot{\boldsymbol{g}}_{T}\right\|}{\mu\left|\lambda_{N}\right|+r\left\|\dot{\boldsymbol{g}}_{T}\right\|} \frac{\dot{\boldsymbol{g}}_{T}}{\left\|\dot{\boldsymbol{g}}_{T}\right\|} \mu\left|\lambda_{N}\right| \\
& =\frac{\boldsymbol{\lambda}_{T}-r \dot{\boldsymbol{g}}_{T}}{\left\|\boldsymbol{\lambda}_{T}-r \dot{\boldsymbol{g}}_{T}\right\|} \mu\left|\lambda_{N}\right| \\
& =\operatorname{prox}_{C_{T}\left(\lambda_{N}\right)}\left(\boldsymbol{\lambda}_{T}-r \dot{\boldsymbol{g}}_{T}\right)
\end{aligned}
$$

which means $\left(\dot{\boldsymbol{g}}_{T}, \boldsymbol{\lambda}_{T}\right) \in T_{P}\left(\lambda_{N}\right)$.

\section{EXAMPLES}

In this section, we provide several examples that serve to illustrate the connections between the LCP and prox formulations graphically and numerically.

\section{A. Point mass on frictional plane}

Is the prox formulation numerically most appropriate? The discussion of this question for a simple planar example is the goal of the following section. The planar restriction is not fundamental. It was imposed only to allow clear graphical illustrations of the solution process.

The instantaneous equations of motion of a point mass in contact with a rigid linear surface (see Fig. 5) can be written as the following system of differential equations:

$$
\begin{aligned}
\dot{q}_{x} & =u_{x}, \\
\dot{q}_{y} & =u_{y}, \\
m \dot{u}_{x} & =h_{x}+\lambda_{T}, \\
m \dot{u}_{y} & =h_{y}+\lambda_{U}
\end{aligned}
$$

where $h_{y}$ is the component of the external force applied to the particle in the direction of the outward normal (the $y$ direction) of the surface and $h_{x}$ is the component tangential to the surface in the positive $x$-direction. Notice also that for this problem, the $x$ - and $y$-coordinates of the particle are the displacement functions, $q_{x}=g_{T}$ and $q_{y}=g_{U}$. To formulate the dynamics with contact constraints, one can augment the differential equations with contact conditions represented at 


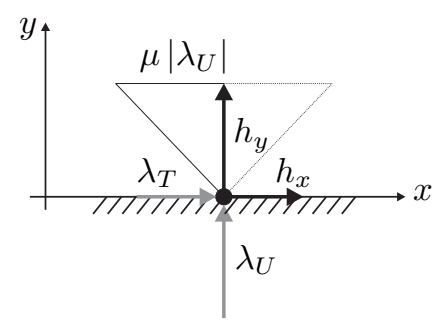

Fig. 5. Configuration of point mass in contact with a surface.

the acceleration level; (5) and (6) for a LCP formulation or $(9 b)$ and $(9 c)$ for a prox formulation:

$$
\begin{aligned}
& \left(\dot{u}_{y}, \lambda_{U}\right) \in U_{C / P}, \\
& \left(\dot{u}_{x}, \lambda_{T}\right) \in T_{C / P}\left(\lambda_{U}\right)
\end{aligned}
$$

where $\dot{u}_{y}=\ddot{g}_{U}$ and $\dot{u}_{x}=\ddot{g}_{T}$.

\section{Fixed-point iteration and prox formulation}

Substituting (21) and (22) into the right-hand-sides of (9b) and $(9 c)$ (at the acceleration level) yields the fixed-point equations:

$$
\begin{aligned}
& \lambda_{U}=\operatorname{prox}_{C_{U}}\left(\left(1-\frac{r}{m}\right) \lambda_{U}-\frac{r}{m} h_{y}\right), \\
& \lambda_{T}=\operatorname{prox}_{C_{T}\left(\lambda_{U}\right)}\left(\left(1-\frac{r}{m}\right) \lambda_{T}-\frac{r}{m} h_{x}\right) .
\end{aligned}
$$

The simultaneous solution of these equations yields the contact force and the acceleration of the particle. Notice that the first equation is completely independent of the second, but the second is coupled to the first through the set $C_{T}\left(\lambda_{U}\right)$.

Consider the problem of finding the normal component of the contact force for when the particle is in contact with the plane $\left(q_{y}=0\right)$ with zero normal velocity $\left(u_{y}=0\right)$ and with the external force pushing the particle against the plane. Fig. 6 illustrates the progress of the fixed-point iterations (dashed curve) with $r$ in the range $0<r<2 m$, starting from an (infeasible) initial guess $\lambda_{U}^{0}$ to the unique solution $\lambda_{U}^{*}=-h_{y}$. Graphically, each iteration consists of two steps: first project the current solution estimate $\lambda_{U}^{k}$ vertically to the prox function (dotted curve), then project horizontally from that point to the line with unit slope, $f\left(\lambda_{U}\right)=\lambda_{U}$. In this picture, as $r$ is varied, the slope of the non-constant part of the prox function changes while the intersection point (i.e. the solution) remains at $\lambda_{U}^{*}=-h_{y}$. Notice that when $r=m$, the prox function becomes a horizontal line and only one iteration is needed to find the solution. The other case of interest for the normal contact force problem arises when the external force acts to separate the particle from the plane (i.e. $h_{y}>0$ ). In this case, the prox function and the line of unit slope have a unique intersection point at $\lambda_{U}^{*}=0$, to which the iterates converge if $0<r<2 m$.

Next consider the tangential subproblem of the particleplane system where the particle is on the plane and sticking (i.e. $g_{U}=0, \dot{g}_{U}=0$ and $\dot{g}_{T}=0$ ) at the current time instant. Assume that the external force acting on the particle has its normal component towards the rigid surface (i.e. $h_{y}<0$ ). Further assume that the solution $\lambda_{U}^{*}=-h_{y}$

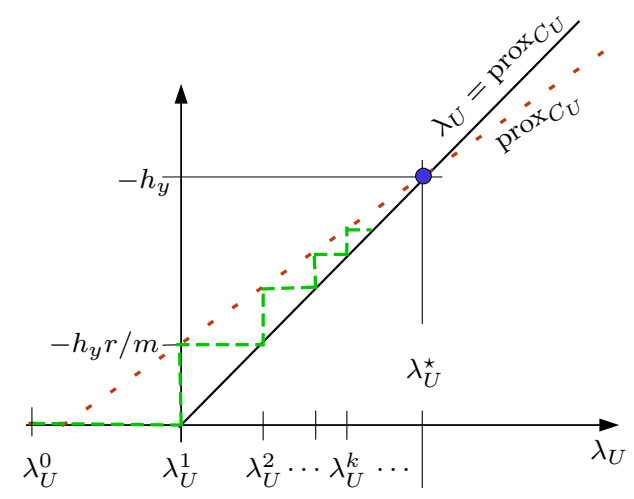

Fig. 6. Progression of iterates for finding the normal contact force.

is already known. Then, the prox formulation is given as: $\lambda_{T}=\operatorname{prox}_{C_{T}\left(\lambda_{U}\right)}\left(\lambda_{T}(1-r / m)-h_{x} r / m\right)$. Fig. 7 shows the prox function (dotted curve), the line of unit slope and the progress of the fixed-point iterations (dashed curve) for $0<h_{x}<\mu\left|h_{y}\right|$ and $m<r<2 m$. Notice that a unique solution exists, $\lambda_{T}^{*}=h_{x}$, and that the iterates converge for any initial guess for $\lambda_{T}$.

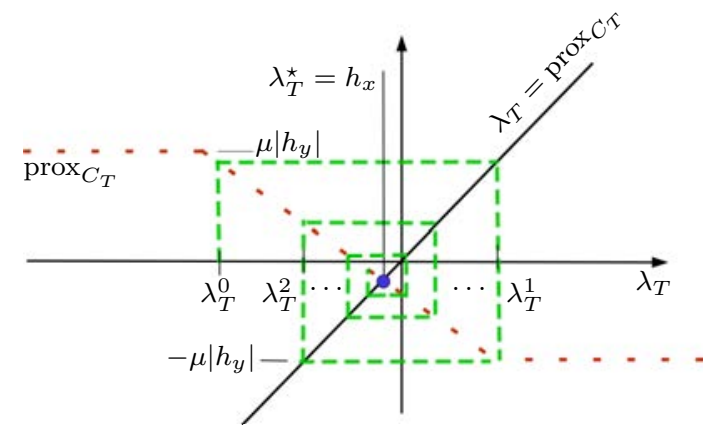

Fig. 7. Progression of iterates for finding the tangential contact force.

In practice, the numerical solution proceeds by interleaving fixed-point iterations between the normal and tangential prox functions. For the particle-on-plane problem, the graph in Fig. 6 is unchanged, hence the normal force iterations are unchanged. However, the graph in Fig. 7 is dependent on the result of the preceding iteration for the normal force. The tangential iterations are guaranteed to converge to the correct value as the normal direction iterations converge, because the iterations for the tangential component converge from any starting guess.

To summarize despite the trivial nature of this system, the analysis suggests that solving multibody dynamics timestepping subproblems by fixed-point iteration of the prox formulation requires one to be careful about the choice of the parameter $r$. At this point, it is not clear how to choose $r$ to guarantee convergence for general problems. We conjecture that the choices of $r$ yielding global convergence to a physically correct solution of the time-stepping subproblem are likely to be related to eigenvalues of the Delassus matrix of the contact configuration [8]. 


\section{Complementarity formulations at the acceleration level}

Let us replace $\lambda_{T}$ with the sum of its positive and negative parts: $\lambda_{T}=\lambda_{f 1}-\lambda_{f 2}$ where $\lambda_{f 1} \geq 0$ is the friction force component acting in the positive $x$-direction and $\lambda_{f 2} \geq 0$ acts in the $(-x)$-direction. Now, the equations of motion (2124) become linear in the unknown forces, accelerations and in the auxiliary variable $\sigma$ :

$$
\begin{aligned}
& m \dot{u}_{x}=h_{x}+\lambda_{f 1}-\lambda_{f 2}, \\
& m \dot{u}_{y}=h_{y}+\lambda_{U} \\
& 0 \leq \lambda_{U} \perp \dot{u}_{y} \geq 0 \\
& 0 \leq \lambda_{f 1} \perp \dot{u}_{x}+\sigma \geq 0 \\
& 0 \leq \lambda_{f 2} \perp-\dot{u}_{x}+\sigma \geq 0 \\
& 0 \leq \sigma \perp \mu \lambda_{U}-\lambda_{f 1}-\lambda_{f 2} \geq 0 .
\end{aligned}
$$

Also at a solution, we have $\sigma=\left|\dot{u}_{x}\right|$ [5]. The above mixed LCP can be reformulated as a standard LCP of size four by eliminating $\dot{u}_{x}$ and $\dot{u}_{y}$.

The numerical solver has to test pivots until a solution is found. In the worst case, this is $2^{n}$ pivots where $n$ is the number of unknowns. However, the average time required by Lemke's algorithm is approximately cubic in $n$.

\section{B. PAinlevé's paradox}

PAINLEVÉ's paradox refers to problems in multibody dynamics in which the solution of the instantaneous dynamics is not unique. One such "paradox" arises in the planar case

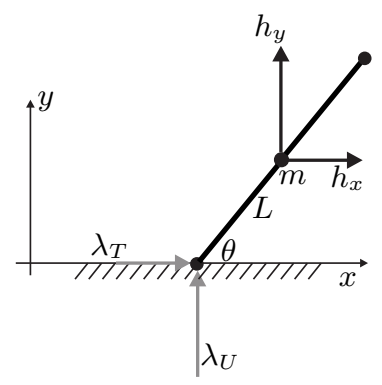

Fig. 8. PAINLEvé problem with $g>0$.

of a rigid object translating in contact with a rigid horizontal surface. The number of solutions of the acceleration-level formulation depends on the values of the mass, moment of inertia, coefficient of friction, and the angle and distance from the contact point to the center of mass of the object (see Fig. 8). In the case considered here, a gravitational force is assumed to act downward through the center of mass perpendicular to the contact tangent, i.e. $h_{y}=-m g$. Since $m$ and $g$ are positive, $h_{y}$ is negative. The lateral external force $h_{x}$ is zero.
The dynamic equations are:

$$
\begin{aligned}
\dot{q}_{x} & =u_{x}, \\
\dot{q}_{y} & =u_{y}, \\
\dot{q}_{\theta} & =u_{\theta}, \\
m \dot{u}_{x} & =\lambda_{T} \\
m \dot{u}_{y} & =\lambda_{U}+h_{y}, \\
J \dot{u}_{\theta} & =\frac{L}{2}\left(\lambda_{T} \sin \left(q_{\theta}\right)-\lambda_{U} \cos \left(q_{\theta}\right)\right)
\end{aligned}
$$

where $J$ is the moment of inertia about an axis perpendicular to the plane of motion and through the mass center. $L / 2$ is the distance from the contact point to the center of mass.

Let us assume that at the current time instant, the object is translating toward the left and leaning toward the right (i.e. $u_{x}<0, u_{y}=u_{\theta}=0$ and $0<q_{\theta}<\pi / 2$ ). The direction of the friction force is determined from velocity kinematics to be in the positive $x$-direction, because the contact point is initially sliding. The unilateral contact constraint must still be evaluated at the acceleration level because $u_{y}=0$ and $\dot{u}_{y}=0$. Thus, the contact constraints for this problem are:

$$
\begin{aligned}
& \left(\ddot{g}_{U}, \lambda_{U}\right) \in U_{C / P}, \\
& \left(\dot{g}_{T}, \lambda_{T}\right) \in T_{C / P}\left(\lambda_{U}\right) .
\end{aligned}
$$

The kinematic quantities, $g_{U}$ and $g_{T}$, and their relevant time derivatives are:

$$
\begin{array}{r}
g_{T}=q_{x}-\frac{L}{2} \cos \left(q_{\theta}\right), \\
g_{U}=q_{y}-\frac{L}{2} \sin \left(q_{\theta}\right), \\
\dot{g}_{T}=u_{x}+\frac{L}{2} u_{\theta} \sin \left(q_{\theta}\right), \\
\ddot{g}_{U}=\dot{u}_{y}+\frac{L}{2}\left(u_{\theta}^{2} \sin \left(q_{\theta}\right)-\dot{u}_{\theta} \cos \left(q_{\theta}\right)\right) .
\end{array}
$$

Note that (40) with $\dot{u}_{x}<0$ implies that $\lambda_{T}=\mu \lambda_{U}$. This allows us to reduce the formulation of the problem to an LCP of size one:

$$
0 \leq \lambda_{U} \perp G \lambda_{U}+b \geq 0
$$

where $G=\frac{1}{m}+\frac{L^{2} \cos \left(q_{\theta}\right)}{4 J}\left(\cos \left(q_{\theta}\right)-\mu \sin \left(q_{\theta}\right)\right)$ and $b=-g$. Note that for physically plausible values of $m, J, L, q_{\theta}$, and $\mu, G$ can be made negative. By choosing the sign of the gravitational acceleration, $b$ can be made negative (the force acts downward) or positive (the force acts upward). For this problem, it is known that when $G$ is strictly positive (Figs. 9 and 10), there is a unique solution for any $b$ [9]. Also if $G$ is strictly negative then if $b$ is strictly positive, there are two solutions (Fig. 12), and if $b$ is strictly negative (Fig. 11), there is no solution.

Using the initial conditions, the prox formulation can be written as a single scalar fixed-point problem:

$$
\lambda_{U}-\operatorname{prox}_{C_{U}}\left(\lambda_{U}-r \ddot{g}_{U}\right)=0 .
$$

Figs. 9-12 show that there is a unique solution when $G>0$ and either zero or two solutions when $G<0$ by intersecting the prox function (dotted curve) with the line of unit slope. 


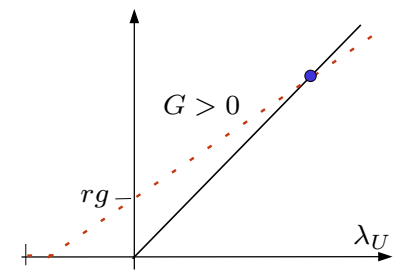

Fig. 9. Globally convergent unique solution: $g, G>0$. Contact is maintained.

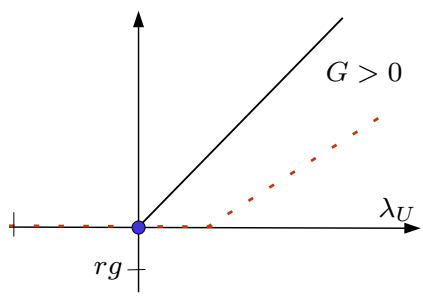

Fig. 10. Globally convergent unique solution: $g<0, G>0$. Contact is lost.

From the point of view of solution existence, the prox formulation completely agrees with complementarity theory. A difference appears when attempting to find a solution via the fixed-point iteration scheme. In the case with $g$ and $G$ negative, for any initial guess $\lambda_{U}^{0}$ less than the second solution $\left(\lambda_{U}^{*}\right)_{2}$, the iterates will converge to the first solution $\left(\lambda_{U}^{*}\right)_{1}=0$. Iterations beginning with $\lambda_{U}^{0}>\left(\lambda_{U}^{*}\right)_{2}$ will diverge. While a pivoting algorithm solving the LCP formulation could terminate at either solution (or be modified to find both), the fixed-point scheme will only find $\left(\lambda_{U}^{*}\right)_{1}$. Another interesting difference is that in the case with $g$ positive and $G$ negative, fixed-point iterations are divergent from any starting guess. Thus, one sees that a pivoting method is superior in that it will be able to recognize the case of solution non-existence. Whereas, the fixed-point scheme will not be able to determine if divergence is due to a poor initial guess or solution non-existence.

\section{CONCLUSIONS AND FUTURE WORKS}

In this paper, we have shown the equivalence of two popular formulations of nonsmooth dynamic systems. Prox formulations lend themselves to solution by fixed-point iteration or root-finding schemes for nonsmooth equations. Complementarity formulation can be approached in a number of ways including pivoting methods. While some have reported faster solution times with fixed-point schemes, the simple problems discussed here shows that fixed-point iterations can diverge when a solution exists and that this solution will be found by a pivoting scheme. However, fixed-point schemes are worth pursuing further to explore the exploitation of finegrained parallelism in the solution process. While regularization of complementarity formulations have allowed one to exploit parallel implementation of quadratic program solvers, they do so at the expense of the introduction of a-physical effects. Parallel implementations of fixed-point schemes will not require such model adjustments. For the analysis, general

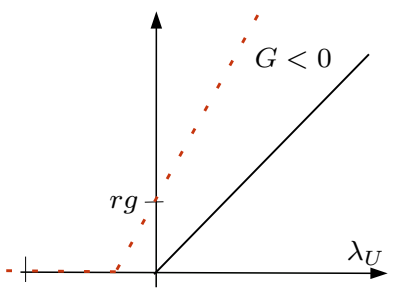

Fig. 11. No solution exists: $g>0, G<0$. The acceleration-based model fails.

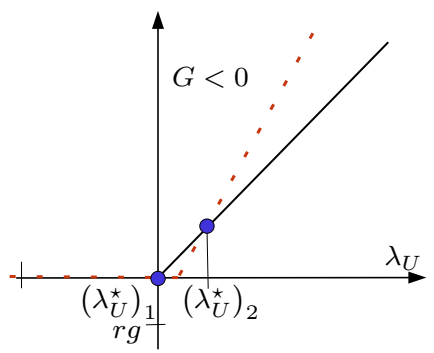

Fig. 12. Two solutions exist: $g, G<0$. Fixed-point iterations can diverge.

approaches will have to be worked out in detail and applied to use case examples.

\section{REFERENCES}

[1] P. Alart and A. Curnier, "A mixed formulation for frictional contact problems prone to Newton like solution methods," Comput Methods Appl Mech Engrg, vol. 92, pp. 353 - 375, 1991.

[2] F. Pfeiffer, Mechanical system dynamics, corr. 2nd printing ed., ser. Lecture notes in applied and computational mechanics. Berlin: Springer, 2008, vol. 40.

[3] A. Transeth, R. Leine, C. Glocker, and K. Pettersen, "3-D snake robot motion: nonsmooth modeling, simulations, and experiments," IEEE Trans Robot, vol. 24, pp. 361-376, 2008.

[4] V. Acary, O. Bonnefon, and B. Brogliato, Nonsmooth modeling and simulation for switched circuits, ser. Lecture Notes in Electrical Engineering. Dordrecht: Springer, 2011, vol. 69.

[5] J. Trinkle, J.-S. Pang, S. Sudarsky, and G. Lo, "On dynamic multi-rigidbody contact problems with Coulomb friction," J Appl Math Mech, vol. 4, pp. 267-279, 1997.

[6] M. Ferris and T. Munson, "Interfaces to PATH 3.0: design, implementation and usage," Comput Optim Appl, vol. 12, pp. 207-227, 1999.

[7] V. Acary, B. Brogliato, A. Daniilidis, and C. Lemarechal, "On the equivalence between complementarity systems, projected systems and unilateral differential inclusions," INRIA, Tech. Rep., 2004.

[8] M. Förg, Mehrkörpersysteme mit mengenwertigen Kraftgesetzen : Theorie und Numerik, als Manuskript gedruckt ed., ser. Fortschritt-Berichte VDI : Reihe 20, Rechnerunterstützte Verfahren. Düsseldorf: VDI Verlag, 2007, vol. 411.

[9] R. Cottle, The linear complementarity problem, ser. Classics in applied mathematics ; 60, J.-S. Pang and R. Stone, Eds. Philadelphia: SIAM, 2009. 